IAU Colloquium 190 on Magnetic Cataclysmic Variables

ASP Conference Series, Vol. 315, 2004

Sonja Vrielmann 89 Mark Cropper, eds.

\title{
H $\alpha$ Doppler Tomography of AM Her: Evidence for Bipolar Accretion
}

\author{
Christos Papadimitriou ${ }^{1,2}$, Emilios Harlaftis ${ }^{2}$ \\ ${ }^{1}$ Department of Astrophysics, Astronomy and Mechanics, University of \\ Athens, 15784 Zografos, Athens, Greece \\ ${ }^{2}$ Institute of Astronomy and Astrophysics, National Observatory of \\ Athens, P. O. Box 20048, Athens 118 10, Greece
}

\begin{abstract}
We present Doppler maps of Halpha emission lines of AM Her from four differents epochs. The tomography shows a strong velocity "spot" emission at $(-100,-100) \mathrm{km} \mathrm{s}^{-1}$ and a weaker "spot" emission at $(-100,-400) \mathrm{km} \mathrm{s}^{-1}$ which is suggestive of bi-polar accretion onto the white dwarf with stronger emission from the main pole at phase $\phi=0.0$.
\end{abstract}

\section{Introduction}

The emission lines of AM Her consist of apparently two components, a broad and a narrow component which have been related to the gas stream and donor star emission, respectively (Liebert and Stockman 1985). The long-term photometric light curve shows low and high states and therefore we embarked on a monitoring campaign in order to search for correlations between the emission line components and the optical/X-ray continuum level. The phase-resolved observations of AM Her were carried out with the $2.5 \mathrm{~m}$ Isaac Newton Telescope (INT) on 1989 July 21 (first epoch, $V \approx 14.4$ ), on 1992 May 17 (second epoch, $\mathrm{V} \approx 14.5$ ), on 1995 July 3 (third epoch, $\mathrm{V} \approx 13.2$ ) and on 1995 July 27 (fourth epoch, $\mathrm{V} \approx 12.9$ ). We obtained $44,31,30,24$ spectra covering 1 orbital cycle at a resolution of $32,44,76,76 \mathrm{~km} \mathrm{~s}^{-1}$, respectively. The CCD images were reduced and the spectra extracted using the optimal extraction technique (Marsh 1989). The spectra were assigned orbital phases using the ephemeris HJD = $2443014.76614+0.128927041 \times E$ (Heise \& Verbunt 1988).

\section{Results}

Image reconstruction was performed on the spectra using the linear, Fourierfiltered back projection technique (Horne 1991). The Doppler velocity maps show that the dominant emission rises from the $\left(-V_{x},-V_{y}\right)$ velocity quadrant in which the gas stream velocities lie. However, it is notable that this strong "spot" emission is of low velocity, $(-100,-100) \mathrm{km} \mathrm{s}^{-1}$, persists in all four epochs, corresponds to the narrow, low velocity ' $\mathrm{S}$ '-wave component on the trailed spectra and is not directly related to the gas stream ballistic trajectory. Such emission is also seen in the UV (SiIV $1394 \AA$, CIII $1176 \AA$ ) Doppler maps (Gänsicke et al. 1998) but in contrast to a HeII Doppler map of AM Her which shows "quasi-ballistic" gas stream emission (Schwarz et al. 2002). However, our Doppler maps show, in 

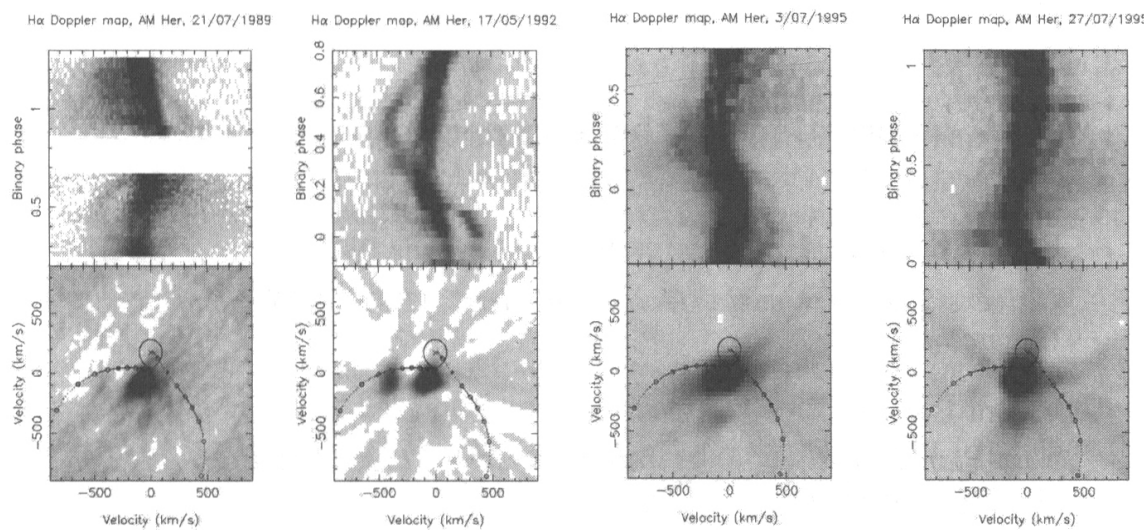

Figure 1. The Ha trailed spectra and Doppler maps of AM Her at four epochs. See text for interpretation. Binary parameters used for the Roche lobe and ballistic trajectory are $K_{2}=174 \mathrm{~km} \mathrm{~s}^{-1}$ and $q=0.7$ (low mass white dwarf).

addition, a weaker "spot" emision at $(-100,-400) \mathrm{km} \mathrm{s}^{-1}$ which is detectable in three epochs. This is compatible with bi-polar accretion (but not with the magnetic-propeller model, Wynn et al. 1997) with the emission from the main accretion pole (phase 0.0 ) much stronger than the secondary pole (phase 0.5 ). During one epoch (low state), $\mathrm{H} \alpha$ emission along the ballistic trajectory is detectable at 0.4-0.5 $\mathrm{R}_{L_{1}}$ which may well signify the impact of the gas stream on the Alfven radius of the magnetosphere.

Acknowledgments CP acknowledges partial financial support received from IAU to take part in the IAU Colloquium on MCVs.

\section{References}

Heise, J. \& Verbunt, F., 1988, A\&A, 189, 112

Liebert, J. \& Stockman, H. S. 1985, in $C V s$ and $L M X B s$, eds D.Q. Lamb, J. Patterson. Dordrecht and D. Reidel Publishing Co., 151

Marsh, T. 1989, PASP, 101, 1032

Horne, K., 1991, in: Fundamental Properties of Cataclysmic Variable Stars: 12th North American Workshop on Cataclysmic Variables and Low Mass X-ray Binaries, San Diego State University Publication, San Diego, ed. A.W. Shafter, 23

Gänsicke, B.T., Hoard, D.W., Beuermann, K., Sion, E.M., \& Szkody, P. 1998, A\&A, 338, 933

Schwarz, R., Hedelt, P., Rau, A., Staude, A., \& Schwope, A.D., 2002, in: The Physics of Cataclysmic Variables and Related Objects, edited by B.T.

Gansicke, K. Beuermann, and K. Reinsch, ASP Vol. 261, 167

Wynn, G.A., King, A.R., \& Horne, K. 1997, MNRAS, 286, 436 